\title{
Multiple Anesthesia/Surgery Cannot Impair Reference Memory in Adult Mice
}

\author{
Xiaoxin Zhou $\mathbb{D}^{1},{ }^{1}$ Jian Lu $\left(\mathbb{D},{ }^{1,2}\right.$ Tong Wu $\mathbb{D}^{1},{ }^{1}$ Xuliang Jiang $\mathbb{D}^{1},{ }^{1}$ Weitian Tian $\mathbb{D}^{1}{ }^{1}$ \\ Wanbing Dai $\mathbb{D}^{1},{ }^{1}$ Siyi $Q i \mathbb{D}^{1},{ }^{1}$ Xuemei Chen $\mathbb{D}^{1},{ }^{1}$ Jiaqiang Zhang $\mathbb{D}^{3},{ }^{3}$ and Diansan Su $\mathbb{D}^{1}$ \\ ${ }^{1}$ Department of Anesthesiology, Renji Hospital, Shanghai Jiaotong University, School of Medicine, Shanghai, China \\ ${ }^{2}$ Department of Anesthesiology, The Second Affiliated Hospital of Jiaxing University, Jiaxing City, Zhejiang Province, China \\ ${ }^{3}$ Department of Anesthesiology and Perioperative Medicine, Henan Provincial People's Hospital, People's Hospital of \\ Zhengzhou University, Zhengzhou, Henan Province, China
}

Correspondence should be addressed to Diansan Su; diansansu@yahoo.com

Xiaoxin Zhou and Jian Lu contributed equally to this work.

Received 26 December 2019; Revised 16 February 2020; Accepted 18 February 2020; Published 7 March 2020

Academic Editor: Carla Sipert

Copyright ( 2020 Xiaoxin Zhou et al. This is an open access article distributed under the Creative Commons Attribution License, which permits unrestricted use, distribution, and reproduction in any medium, provided the original work is properly cited.

\begin{abstract}
Postoperative cognitive dysfunction increases mortality and morbidity in perioperative patients. Numerous studies have demonstrated that multiple surgery/anesthesia during the neurodevelopmental period affects cognitive function, whereas a single anesthesia/surgery rarely causes cognitive dysfunction in adults. However, whether adults who undergo multiple anesthesia/surgery over a short period will experience cognitive dysfunction remains unclear. In this study, central nervous system inflammation and changes in cholinergic markers were investigated in adult mice subjected to multiple laparotomy procedures over a short period of time. The results showed that despite the increased expression of IL- 6 and TNF- $\alpha$ in the hippocampus after multiple operations and the activation of microglia, multiple anesthesia/surgery did not cause a decline in cognitive function in adult mice. There were no changes in the cholinergic markers after multiple anesthesia/surgery.
\end{abstract}

\section{Introduction}

Postoperative cognitive dysfunction (POCD) is a common complication after a major surgery [1-3]. The condition is characterized by impaired learning and memory and may persist for months and years after surgery $[4,5]$. POCD is thought to be associated with major surgery and advanced age $[6,7]$, and there is no doubt that older individuals are more likely to develop POCD [8]. Although POCD does occur in adults in clinical settings, whether adult mice develop similar learning and memory impairment after anesthesia/surgery remains controversial.

Lin and Zuo demonstrated that 4-month-old male rats exposed to isoflurane had significant impairments in longterm spatial memory assessed using a Barnes maze in addition to impaired hippocampus-dependent learning and memory in a fear conditioning test [9]. However, our previous work showed that single anesthesia/surgery induced memory decline and attenuated central cholinergic biomarkers in aged mice but not in adult mice [10]. Walters et al. [11] demonstrated that exposure to anesthesia alone does not cause persistent learning and memory impairments in adult monkeys. Nearly all studies on adult animals have focused on single anesthesia/surgery, and few have explored what happens to adult mice after multiple anesthesia/surgery. In the present study, we hypothesized that multiple anesthesia/surgery would impair the reference memory in adult mice.

\section{Materials and Methods}

Ethical approval for this study was provided by the Animal Care and Use Committee of Shanghai Jiao Tong University, School of Medicine. All animal procedures were performed in accordance with the National Institutes of Health animal care guidelines. 
2.1. Animals and Anesthesia/Surgery Procedure. C57BL/6J mice (aged 8 weeks, male) were purchased from the Animal Research Center of Shanghai Jiaotong University, School of Medicine. The animals were housed in standard cages (size, $325 \times 210 \times 180 \mathrm{~mm}, 4-5$ mice per cage) under controlled laboratory conditions (temperature of $22 \pm 2{ }^{\circ} \mathrm{C}, 12 \mathrm{~h}$ light $/ 12 \mathrm{~h}$ dark cycle) with free access to regular rodent pellets and water. All mice were allowed to adapt to their new environment for 7 days before beginning the experiments.

The mice were randomly divided into three groups: control group, single anesthesia/surgery group, and multiple anesthesia/surgery group. Exploratory laparotomy was performed under isoflurane anesthesia (induced with $4.0 \%$ isoflurane and maintained with $2.0 \%$ isoflurane in 0.30 $\mathrm{FiO}_{2}$ ). The mice were gently restrained to a heating pad $\left(37^{\circ} \mathrm{C}\right)$ using paper tape, and the whole procedure lasted $10 \mathrm{~min}$. Mice in the single anesthesia/surgery group received surgery once, and those in the multiple anesthesia/surgery group underwent surgery every four days (3 total operations) (Figure 1(a)).

Spatial reference memory in mice from the control and the multiple anesthesia/surgery groups was evaluated in the Morris water maze (MWM) since the second day after the last surgery. In different cohorts of animals, 8 mice from control, single anesthesia/surgery, and multiple anesthesia/surgery groups were euthanized at each time point $(6,24$, and $48 \mathrm{~h}$ after surgery) in each group. Hippocampus tissue and serum were harvested for the measurements of IL- $1 \beta$, IL-6, TNF- $\alpha$, and IL-10 by enzyme-linked immunosorbent assay (ELISA). Hippocampus tissue was dissected for detecting cholinergic markers using western blot analysis, and the whole brain was dissected for microglial immunofluorescence staining.

2.2. Morris Water Maze (MWM). The MWM test was performed using a computerized video tracking system as previously described [12], and the method description partly reproduces their wording. For the reference memory test, animals were maintained in the same rearing conditions throughout the procedure. The test was performed by an operator blinded to the group conditions. Briefly, a hidden round platform was placed $1 \mathrm{~cm}$ below the water surface in the center of the northeast quadrant of a circular pool $(110 \mathrm{~cm}$ in diameter and $30 \mathrm{~cm}$ in depth). The water was maintained at $23-25^{\circ} \mathrm{C}$, and the pool was situated in a room with visual cues. The position of the cues remained unchanged throughout the task.

Before the anesthesia/surgical procedure, mice were individually handled for 2 min each day for 1 week. After the anesthesia/surgical procedure, training was conducted for 5 days for the reference memory test (Figure 1(a)). The platform was located in the center of the fourth quadrant. In all trials, the mouse was released into the water facing the pool wall from one of four separate quadrants and allowed to swim until it climbed on to the platform. Once the mouse found the platform, the trial was terminated, and the mouse was allowed to stay on the platform for $15 \mathrm{~s}$. If the mouse failed to find the platform within $60 \mathrm{~s}$, it was gently guided to the platform and allowed to remain on the platform for
$15 \mathrm{~s}$. Four trials were conducted every day, separated by a 30-40 min intertrial interval; the platform remained at the same location throughout the test. The amount of time the mouse took to find and mount the platform (escape latency) and the swimming speed were calculated from recorded videos using the MWM software (Shanghai Jiliang Software Technology Co. Ltd., China). The probe test was performed on the fifth day of the reference memory test. In this test, the platform was absent, and the animals were free to swim for $60 \mathrm{~s}$, starting from the quadrant opposite the platform. The times spent in the target and opposite quadrants were recorded.

2.3. Enzyme-Linked Immunosorbent Assay (ELISA) for IL-1 $\beta$, $I L-6, T N F-\alpha$, and $I L-10$. Serum and the hippocampus tissues were collected at indicated time points after surgery in mice from the control, single anesthesia/surgery, and multiple anesthesia/surgery groups. The harvested brain tissues were homogenized on ice using RIPA lysis buffer (Beyotime Biotechnology, Shanghai, China) with protease inhibitors (A32953, Thermo). After collection of the whole blood, it was left undisturbed at room temperature for approximately $30 \mathrm{~min}$. Following centrifugation at $3000 \times \mathrm{g}$ for $10 \mathrm{~min}$ at $4^{\circ} \mathrm{C}$, the supernatant (serum) was collected. Levels of IL- $1 \beta$, TNF- $\alpha$, IL-6, and IL-10 in both serum and hippocampus tissue were measured using mouse ELISA kits (MultiSciences Biotechnology, Hangzhou, China).

2.4. Immunofluorescence Staining. The mice were euthanized and subjected to cardiac perfusion with $20 \mathrm{~mL}$ precooled saline and then with $20 \mathrm{~mL} 4 \%$ paraformaldehyde. The mouse brain was fixed and dehydrated with $20 \%$ and $30 \%$ sucrose solution in turn. The mouse brain was subsequently sectioned into $22 \mu \mathrm{m}$ slices with a freezing microtome (CM3050S, Leica). The brain slices were washed with $0.3 \%$ Triton X-100 in PBS and blocked with blocking buffer (Beyotime Biotechnology, Shanghai, China) for $1 \mathrm{~h}$ at room temperature. The slices were incubated with primary antibody Ibal $(1: 100, \mathrm{Abcam})$ at $4^{\circ} \mathrm{C}$ overnight. The slices were washed with $0.3 \%$ PBST for $30 \mathrm{~min}$ and secondary antibody ( $1: 1000$, Abcam) for $1 \mathrm{~h}$ at room temperature. Fluorescence signals were captured using confocal microscopy (FV3000, Olympus).

2.5. Western Blotting. Brain tissues were harvested, and protein was extracted as previously described [13]. Protein concentration was quantified using bicinchoninic acid [14] (BCA) assay following the manufacturer's instructions (23225, Thermo). Protein can react with alkaline $\mathrm{Cu} 2^{+}$producing cuprous ion $\left(\mathrm{Cul}^{+}\right)$which can form an intense purple complex with bicinchoninic acid in an alkaline environment; the basis of BCA is that the color produced from this reaction increases over a broad range of increasing protein concentrations. Thirty micrograms of protein were separated with $8 \%$ sodium dodecyl sulfate-polyacrylamide gel electrophoresis and transferred to PVDF membranes (Millipore). Nonspecific binding sites were blocked with $1 \%$ bovine serum albumin for $1 \mathrm{~h}$ at room temperature and incubated with choline acetyltransferase (ChAT, 1:1000; Abcam), 


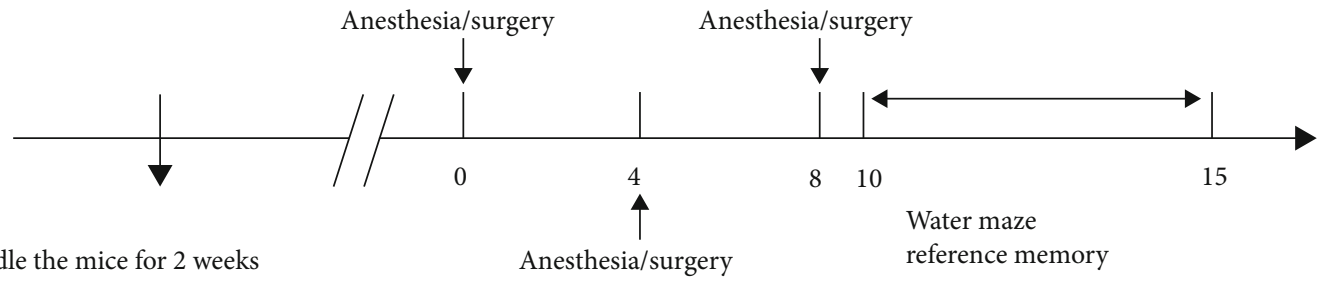

(a)

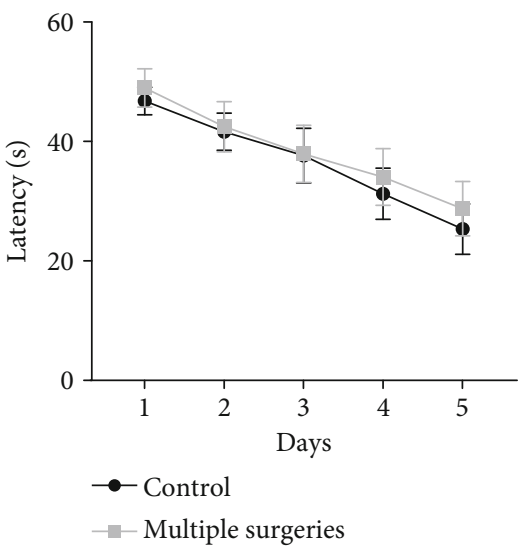

(b)

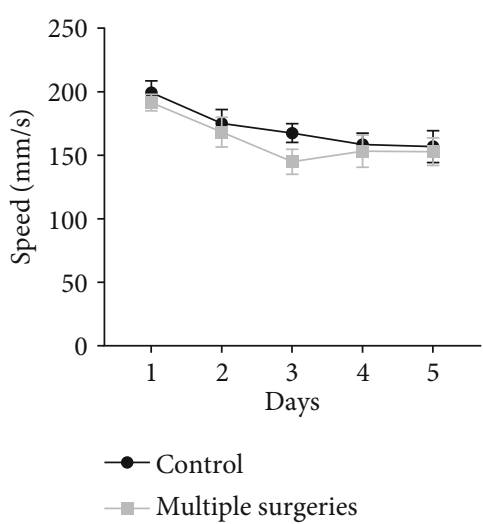

(c)

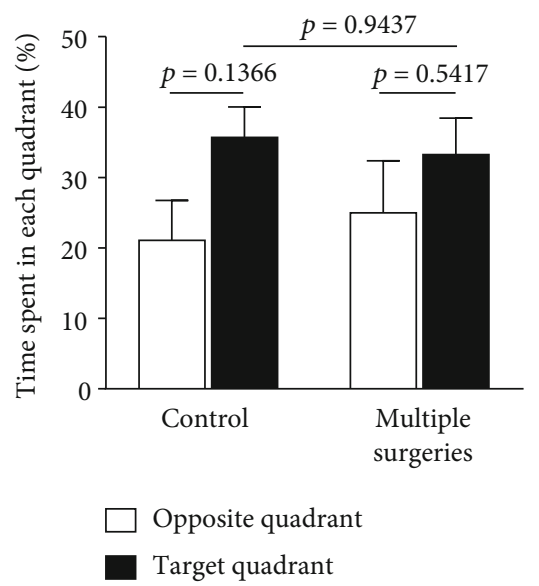

(d)

FiguRE 1: Multiple anesthesia/surgery did not impair spatial reference memory in adult mice. (a) Experimental timeline of surgery procedure and Morris water maze test. Mice in the multiple anesthesia/surgery group underwent surgery every 4 days. After the anesthesia/surgical procedure, training was conducted for 5 days followed by probe tests on day 14. (b) Escape latency to reach the hidden platform during the 5-day training; there was no significant difference between the control group and the multiple surgery group $(p=0.9949)$. (c) Average swimming speed during hidden platform training; there was no significant difference between the control group and the multiple surgery group $(p=0.8907)$. (d) Time spent in the target quadrant during the probe test; there was no significant difference between the control group and the multiple surgery group ( $n=10$ for both control and multiple anesthesia/surgery groups). mA/S: multiple anesthesia/surgery.

acetylcholinesterase (AChE, 1:1000; Abcam), and choline transporter (ChT, 1:1000; Abcam) at $4^{\circ} \mathrm{C}$ overnight. The membranes were washed with $0.1 \%$ TBST for $30 \mathrm{~min}$ and incubated with secondary antibody for $1 \mathrm{~h}$ at room temperature. The membranes were detected using an enhanced chemiluminescence system (Yeasen, China) and analyzed using Image Lab 3.0 (Bio-Rad).

2.6. Statistical Analysis. Statistical analyses were performed with GraphPad Prism 6.0 (GraphPad Software Inc., USA). Data are presented as the mean \pm SEM. A two-way repeated-measures ANOVA was used to analyze the water maze escape latency and average speed. A one-way ANOVA or unpaired $t$ test was used to analyze the probe quadrant trial data, probe test data, abundance of cytokines, relative fluorescence quantification, and relative protein levels of ChAT, AChE, and ChT. Statistical significance was determined if $p<0.05$.

\section{Results}

3.1. Multiple Anesthesia/Surgery Did Not Impair Spatial Reference Memory in Adult Mice. The MWM was used to evaluate learning and memory after three incidences of anesthesia/surgery. After the anesthesia/surgical procedure, training was conducted for 5 days, and probe tests were con- ducted on day 15 (Figure 1(a)). During the training course, the mice from the multiple surgery group did not show longer escape latency compared with the untreated control mice (Figure 1(b)). There was no significant difference in swimming speed between the two groups during the training (Figure 1(c)). During the probe test, the preference for the target quadrant and the time spent looking for the hidden platform were comparable between the control mice and those in the multiple surgery group (Figure 1(d)). These results suggested that multiple anesthesia/surgery does not impair reference memory in young adult mice.

3.2. Single and Multiple Anesthesia/Surgery Induced Transient Neuroinflammation in Different Pattern in Adult Mice. Single anesthesia/surgery induced inflammation in both serum and hippocampus at an early stage ( $6 \mathrm{~h}$ after anesthesia/surgery). As shown in Figure 2, IL-6 in serum increased significantly $6 \mathrm{~h}$ after single anesthesia/surgery and slightly decreased 24 hours later. The level of IL-1 $\beta$, IL-6, and TNF- $\alpha$ in hippocampus increased significantly $6 \mathrm{~h}$ after anesthesia/surgery and reduced to a level comparable to the control group at 24 and $48 \mathrm{~h}$ after surgery.

Multiple anesthesia/surgery induced an early inflammation in serum, but the inflammatory response in the hippocampus occurred relatively late. As shown in 


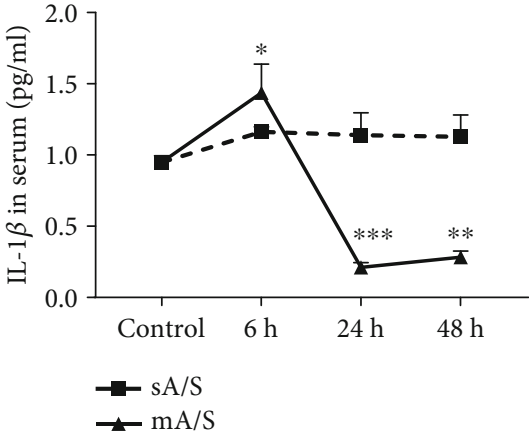

(a)

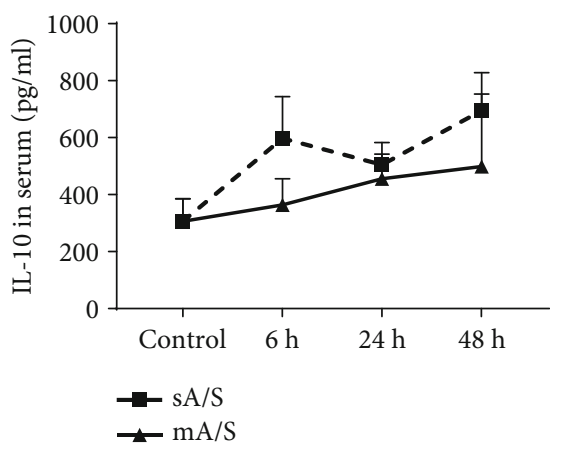

(d)

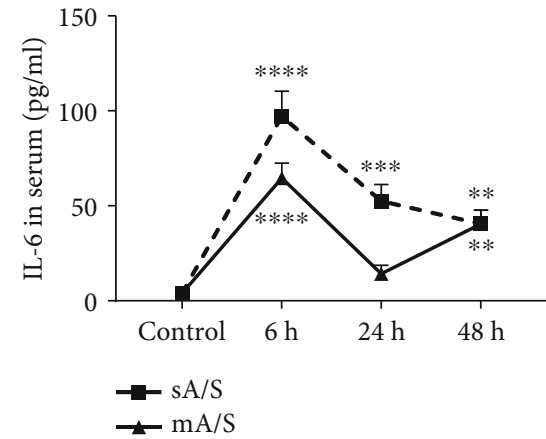

(b)

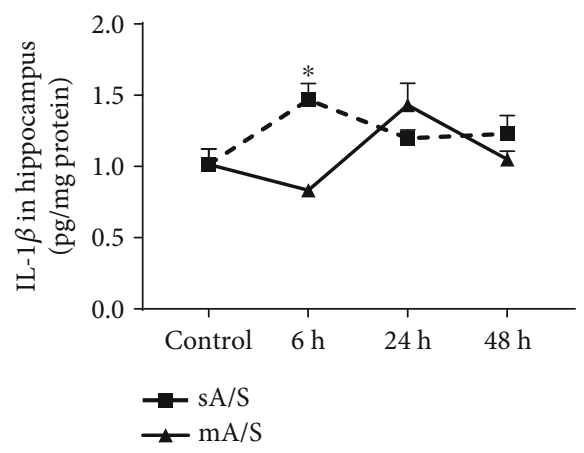

(e)

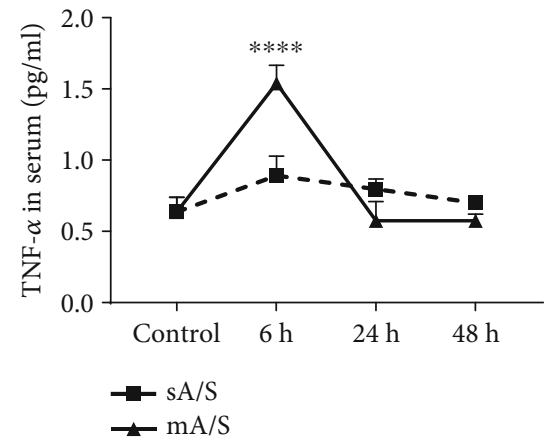

(c)

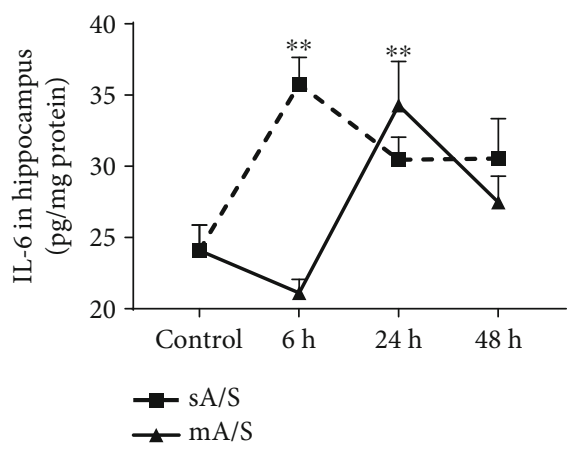

(f)

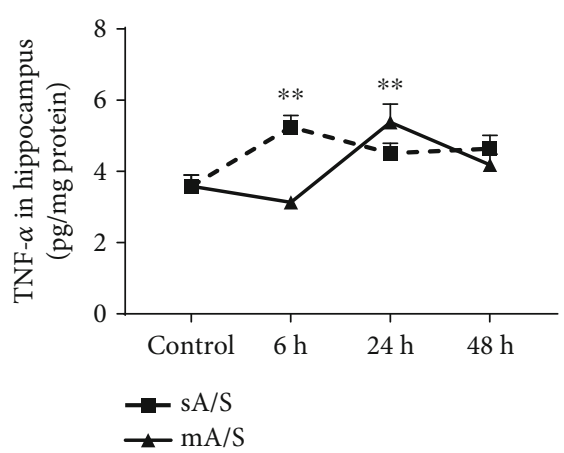

(g)

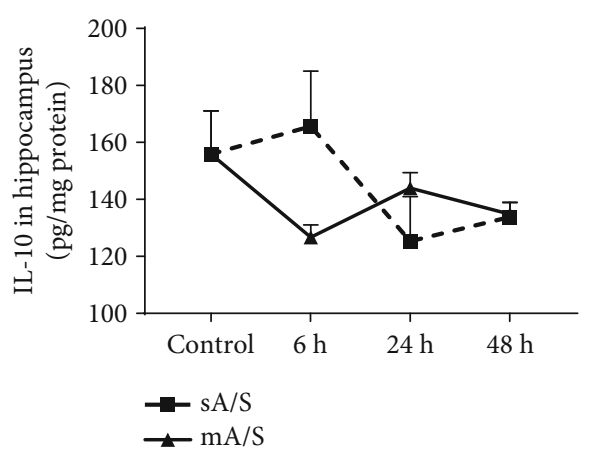

(h)

FIgURE 2: Multiple anesthesia/surgery induced transient central inflammation in hippocampus. (a-c) The levels of IL-1 $\beta$, IL-6, and TNF- $\alpha$ increased in blood at $6 \mathrm{~h}$ after multiple surgeries $\left({ }^{*} p=0.0456\right.$ for IL- $1 \beta,{ }^{* * * *} p=0.0001$ for IL-6, and ${ }^{* * * *} p<0.0001$ for TNF- $\alpha$ ). Only IL-6 increased in blood after single surgery $\left({ }^{* * * *} p=0.0001\right.$ at $6 \mathrm{~h},{ }^{* * *} p=0.003$ at $24 \mathrm{~h}$, and ${ }^{* *} p=0.0096$ at $48 \mathrm{~h}$ ). IL- $1 \beta$ decreased in blood at $24 \mathrm{~h}$ and $48 \mathrm{~h}$ after multiple surgeries $\left({ }^{* * *} p=0.0007\right.$ at $24 \mathrm{~h}$ and ${ }^{* *} p=0.0017$ at $\left.48 \mathrm{~h}\right)$. (e-g) IL-6 and TNF- $\alpha$ increased in the hippocampus at $24 \mathrm{~h}$ after multiple anesthesia/surgery $\left({ }^{* *} p=0.0138\right.$ for IL-6 and ${ }^{* *} p=0.0050$ for TNF- $\left.\alpha\right)$. IL- $1 \beta$, IL-6, and TNF- $\alpha$ increased in the hippocampus at $6 \mathrm{~h}$ after single surgery $\left({ }^{*} p=0.0108\right.$ for IL- $1 \beta,{ }^{* *} p=0.001$ for IL-6, and ${ }^{* *} p=0.0035$ for TNF- $\alpha$ ). (d, h) IL-10 in blood and hippocampus had no significant changes after single/multiple surgeries. Mean (SEM), one-way ANOVA. sA/S: single anesthesia/surgery; $\mathrm{mA} / \mathrm{S}$ : multiple anesthesia/surgery.

Figure 2, IL-1 $\beta$, IL-6, and TNF- $\alpha$ in the serum increased significantly at $6 \mathrm{~h}$ after anesthesia/surgery and then decreased 24 hours later. While IL-6 and TNF- $\alpha$ in hippocampus increased at $24 \mathrm{~h}$ after anesthesia/surgery and decreased at $48 \mathrm{~h}$, both single and multiple anesthesia/surgery did not affect the IL-10 level in serum and hippocampus (Figures 2(d) and 2(h)).

3.3. Both Single and Multiple Anesthesia/Surgery Induced Activation of Microglia in the Hippocampus. The activation of microglia is another reflection of central inflammation.
We stained the postoperative hippocampal section for Iba1, a marker for microglia, at 6,24 , and $48 \mathrm{~h}$ after surgery. The activation of microglia was observed in the single anesthesia/surgery group at all three time points (Figures 3(a) and 3(b)).

3.4. Multiple Anesthesia/Surgery Did Not Impair the Central Cholinergic System. We have previously demonstrated that central cholinergic neuronal degeneration promotes the development of POCD in aged mice [10]. Does multiple anesthesia/surgery affect the central cholinergic system? 

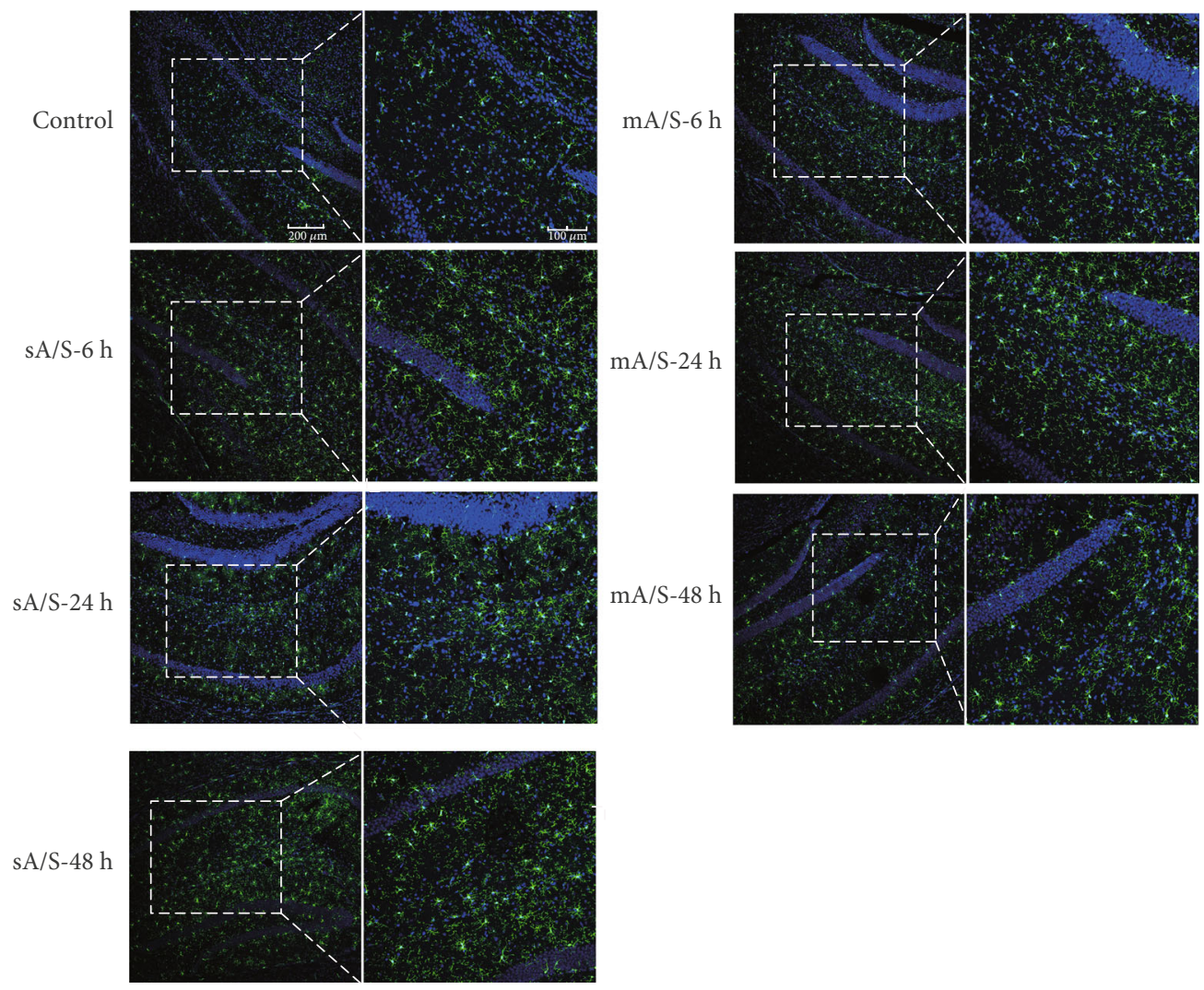

(a)

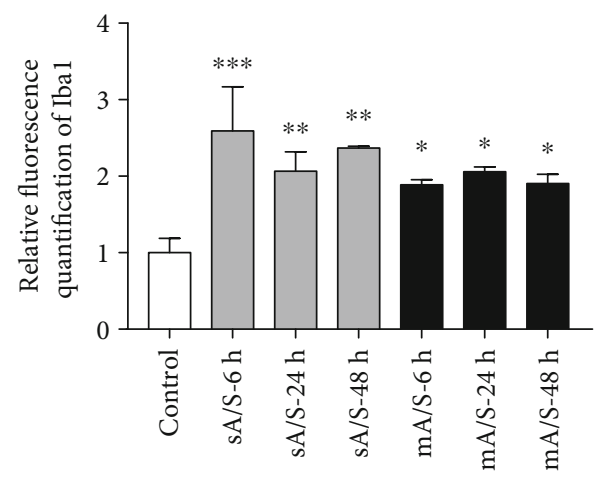

(b)

FIgURE 3: Both single and multiple anesthesia/surgery induced the activation of microglia in the hippocampus. (a) Representative photomicrographs of immunofluorescence of microglia (Iba1, green); nucleus was stained with DAPI (blue). $(n=3-5)$. Scale bar: $200 \mu \mathrm{M}$ (left) and $100 \mu \mathrm{M}$ (right). (b) Fluorescence signal intensity was quantified by ImageJ (versus control, $p=0.0008 \mathrm{for} s \mathrm{~A} / \mathrm{S}-6 \mathrm{~h}, p=0.0139$ for sA/S-24h, $p=0.0034$ for sA/S-48 h, $p=0.0459$ for mA/S-6h, $p=0.0248$ for mA/S-24h, and $p=0.0409$ for mA/S-48 h). Mean (SEM), one-way ANOVA. sA/S: single anesthesia/surgery; $\mathrm{mA} / \mathrm{S}$ : multiple anesthesia/surgery.

The levels of hippocampal ChAT, AChE, and ChT were measured by western blotting at three time points $(6,24$, and $48 \mathrm{~h}$ ) after surgery. No significant change was shown in the central cholinergic system after multiple operations (Figures 4(a)-4(d)).

\section{Discussion}

In the present study, we investigated whether multiple anesthesia/surgery could impair the spatial reference memory of young adult mice and evaluated the changes in neuro- inflammation and the degeneration of central cholinergic neurons after multiple anesthesia/surgery. The results showed that multiple anesthesia/surgery did not impair the spatial reference memory in young adult mice, though the activation of microglia and the increase of proinflammatory cytokines were observed.

Multiple anesthesia/surgery has different effects on the CNS at different developmental stages. Several studies have demonstrated that exposure to multiple anesthesia is neurotoxic to the developing brain [15-18]. However, there has been no study focusing on the effect of multiple 


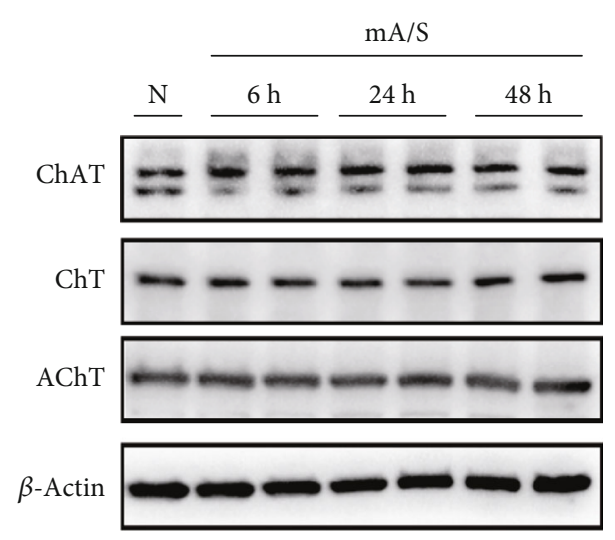

(a)

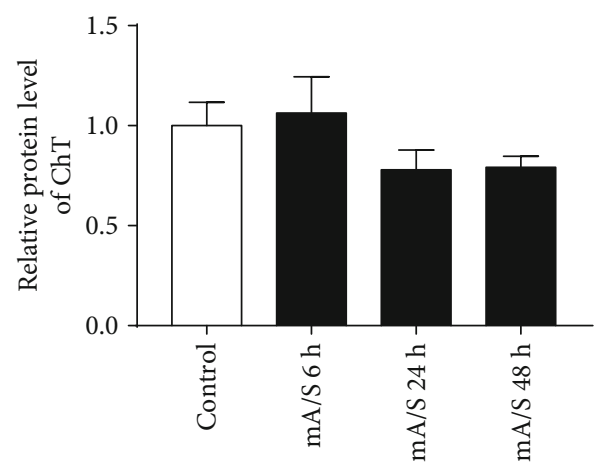

(c)

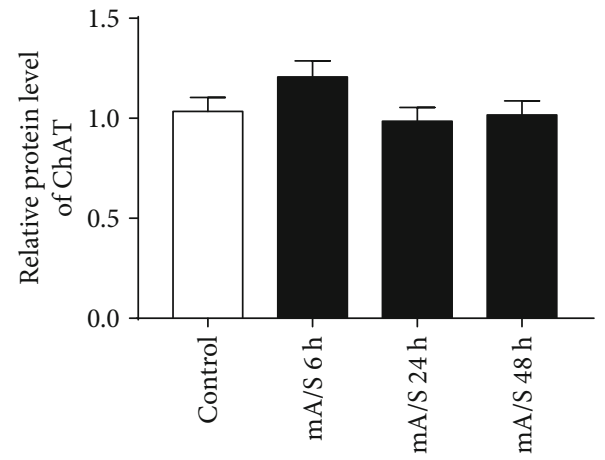

(b)

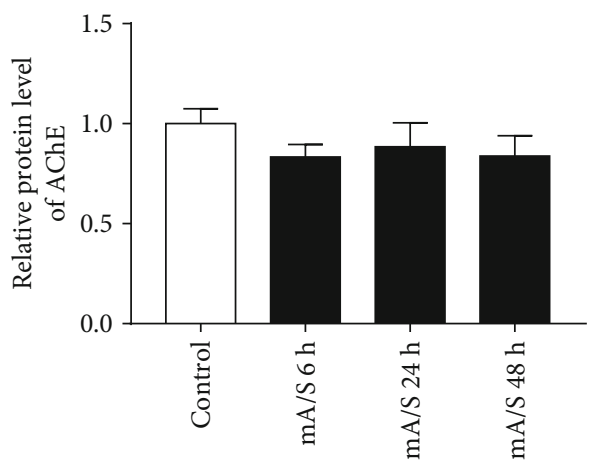

(d)

FIGURE 4: Both single and multiple anesthesia/surgery did not impair the central cholinergic system. (a-d) Representative western blot of ChAT/AChE/ChT proteins and their relative levels in the hippocampus at 6, 24, and 48 hours after surgery. $\beta$-Actin was used for normalizing protein levels. $n=4$ for each group. sA/S: single anesthesia/surgery; mA/S: multiple anesthesia/surgery.

anesthesia/surgery on the adult brain. The results from the present study demonstrated that neuroinflammation induced by multiple anesthesia/surgery is not enough to impair the learning and memory of adult mice.

There are several possible reasons why multiple anesthesia/surgery did not induce the impairment of spatial reference memory in adult mice. (A) The immunological system in adult mice is powerful enough to resolve the neuroinflammation in a very short time. Our results showed that the levels of IL- $1 \beta$, IL- 6 , and TNF- $\alpha$ in the hippocampus elevated at $24 \mathrm{~h}$ after anesthesia/surgery and all returned to a normal range within $48 \mathrm{~h}$. (B) Neuroinflammation was not severe enough after multiple anesthesia/surgery to cause cognitive decline. (C) The cholinergic neuron is strong enough to undergo multiple anesthesia/surgery because no cholinergic markers in the hippocampus were changed despite the repeated damage.

Accumulating evidence shows that neuroinflammation plays a key role in the development of POCD $[19,20]$. The extent of the elevation of proinflammatory cytokines (IL$1 \beta$, IL-6, TNF- $\alpha$, etc.) in both the central nervous system and the systemic circulation after surgery may relate to the degree of cognitive decline [21,22]. These proinflammatory cytokines can be transmitted through the impaired bloodbrain barrier (BBB) to overactivate microglia, resulting in hippocampal neuroinflammation in POCD [23]. The neural system of aged individuals is more susceptible than that of young adults $[24,25]$. Our previous study showed that a single surgery (splenectomy) did not impair the spatial reference memory of young adult mice in spite of the increased IL$1 \beta /$ IL- 6 and activated microglia [26]. The powerful immune system may be helpful in reducing and resolving neuroinflammation after anesthesia/surgery. In addition, Shin et al. demonstrated that the $\mathrm{BBB}$ was damaged in aged mice, but not adult mice after anesthesia/surgery [27].

Immune tolerance might occur after multiple anesthesia/surgery. Wendeln et al. [28] demonstrated that multiple peripheral inflammatory stimuli induced immune tolerance in the brain, which mainly manifested in the elevation of IL-10 in the brain. However, multiple anesthesia/surgery did not induce the elevation of the IL-10 level. Therefore, whether multiple anesthesia/surgery induces immune tolerance in the brain remains unclear. Liu and his colleagues found that neuroinflammation induced by peripheral 
inflammation can be mitigated by the precondition of 3 consecutive intraperitoneal injections of E.coli; the levels of IL$1 \beta$, IL- 6 , and TNF- $\alpha$ in the brain were significantly lower than the controlled group when encountered an acute immune challenge [29]. In the present study, we observed a mild decrease (no statistical differences) in the levels of IL$1 \beta$, IL- 6 , and TNF- $\alpha$ at $6 \mathrm{~h}$ in the hippocampus. Suppose the previous two surgeries were the precondition and the last one surgery was an acute immune challenge, the mild decrease of IL- $1 \beta$, IL- 6 , and TNF- $\alpha$ at $6 \mathrm{~h}$ might result from such preconditioning which activates anti-inflammatory pathway. But the precondition did not affect the activation of microglia, which might explain the delayed increase of IL- 6 and TNF- $\alpha$ (secreted by activated microglia) [30, 31].

Maintaining the central cholinergic system might be another reason why multiple anesthesia/surgery has no effects on spatial reference memory. The central cholinergic system is essential for learning and memory, including the modulation of the acquisition, encoding, consolidation, reconsolidation, extinction, and retrieval of memory [32]. Our previous work showed that central cholinergic neuronal degeneration facilitates the development of POCD [10, 33]. In this study, the central cholinergic system was stable after multiple anesthesia/surgery, corresponding to normal learning and memory.

There are some limitations in this study. We only measured the spatial reference memory, and we observed no changes after multiple anesthesia/surgery. We did not investigate how other domains of learning and memory such as working memory changed after multiple anesthesia/surgery. In our previous study, we demonstrated that although the spatial reference memory was not changed after a single anesthesia/surgery, the working memory was impaired in young adult mice [33]. Although exposure to multiple anesthesia/surgery in such a short period is not common in clinical practice, our data from the present study indicated that the effects of multiple anesthesia/surgery on learning and memory are no worse than those of a single anesthesia/surgery.

In conclusion, in spite of mild neuroinflammation, multiple anesthesia/surgery does not impair spatial reference memory in young adult mice. In clinical settings, patients may require multiple anesthesia/surgery in many situations such as anaplasty after burning or orthopedic deformity correction. The results of the present study indicated that multiple anesthesia/surgery might be not a risk factor for learning and memory impairment after surgery in young adult patients.

\section{Data Availability}

The data used to support the findings of this study are included within the article.

\section{Disclosure}

The funders had no role in the analyses and interpretation of the results or writing of the manuscript.

\section{Conflicts of Interest}

The authors have declared that no competing interests exist regarding the publication of this paper.

\section{Acknowledgments}

This study was supported by grants from the National Natural Science Foundation of China (Nos. 81571030, 81771133, 81970995, 81701092, 81874371, and 81801095), Shanghai Pudong New Area Municipal Commission of Health and Family Planning Funding (PW2016D-4), Shanghai Jiao Tong University Integration Founding of Medicine and Engineering (YG2017MS53), Shanghai Shenkang Hospital Development Center Founding (SHDC12017X11), State Key Laboratory of Neuroscience Funding (SKLN-201803), Renji Hospital Clinical Innovation Foundation (PYMDT-007), Jiaxing Science and Technology Planned Project (2018AY32004), and Shanghai Municipal Education Commission-Gaofeng Clinical Medicine Support (20171916).

\section{References}

[1] M. Berger, J. W. Nadler, J. Browndyke et al., "Postoperative cognitive dysfunction: minding the gaps in our knowledge of a common postoperative complication in the elderly," Anesthesiology Clinics, vol. 33, no. 3, pp. 517-550, 2015.

[2] M. Berger, N. Terrando, S. K. Smith, J. N. Browndyke, M. F. Newman, and J. P. Mathew, "Neurocognitive function after cardiac surgery: from phenotypes to mechanisms," Anesthesiology, vol. 129, no. 4, pp. 829-851, 2018.

[3] H. Abildstrom, L. S. Rasmussen, P. Rentowl et al., "Cognitive dysfunction 1-2 years after non-cardiac surgery in the elderly. ISPOCD group. International Study of Post-Operative Cognitive Dysfunction," Acta Anaesthesiologica Scandinavica, vol. 44, no. 10, pp. 1246-1251, 2000.

[4] F. Holmgaard, A. G. Vedel, L. S. Rasmussen, O. B. Paulson, J. C. Nilsson, and H. B. Ravn, "The association between postoperative cognitive dysfunction and cerebral oximetry during cardiac surgery: a secondary analysis of a randomised trial," British Journal of Anaesthesia, vol. 123, no. 2, pp. 196-205, 2019.

[5] C. Quan, J. Chen, Y. Luo et al., "BIS-guided deep anesthesia decreases short-term postoperative cognitive dysfunction and peripheral inflammation in elderly patients undergoing abdominal surgery," Brain and Behavior: A Cognitive Neuroscience Perspective, vol. 9, no. 4, article e01238, 2019.

[6] S. Glumac, G. Kardum, and N. Karanovic, "Postoperative cognitive decline after cardiac surgery: a narrative review of current knowledge in 2019," Medical Science Monitor, vol. 25, pp. 3262-3270, 2019.

[7] H. Zhang, J. Zheng, R. Wang, G. Wu, and J. Chen, "Serum phosphorylated neurofilament heavy subunit-H, a potential predictive biomarker for postoperative cognitive dysfunction in elderly subjects undergoing hip joint arthroplasty," The Journal of Arthroplasty, vol. 34, no. 8, pp. 1602-1605, 2019.

[8] T. G. Monk, B. C. Weldon, C. W. Garvan et al., "Predictors of cognitive dysfunction after major noncardiac surgery," Anesthesiology, vol. 108, no. 1, pp. 18-30, 2008. 
[9] D. Lin and Z. Zuo, "Isoflurane induces hippocampal cell injury and cognitive impairments in adult rats," Neuropharmacology, vol. 61, no. 8, pp. 1354-1359, 2011.

[10] H. Xu, L. Chen, X. Zhang et al., "Central cholinergic neuronal degeneration promotes the development of postoperative cognitive dysfunction," Laboratory Investigation, vol. 99, no. 7, pp. 1078-1088, 2019.

[11] J. L. Walters, X. Zhang, J. C. Talpos et al., "Sevoflurane exposure has minimal effect on cognitive function and does not alter microglial activation in adult monkeys," Neurotoxicology, vol. 71, pp. 159-167, 2019.

[12] R. Morris, "Developments of a water-maze procedure for studying spatial learning in the rat," Journal of Neuroscience Methods, vol. 11, no. 1, pp. 47-60, 1984.

[13] X. L. Jiang, X. Y. Gu, X. X. Zhou et al., "Intestinal dysbacteriosis mediates the reference memory deficit induced by anaesthesia/surgery in aged mice," Brain, Behavior, and Immunity, vol. 80, pp. 605-615, 2019.

[14] P. K. Smith, R. I. Krohn, G. T. Hermanson et al., "Measurement of protein using bicinchoninic acid," Analytical Biochemistry, vol. 150, no. 1, pp. 76-85, 1985.

[15] J. H. Yon, J. Daniel-Johnson, L. B. Carter, and V. JevtovicTodorovic, "Anesthesia induces neuronal cell death in the developing rat brain via the intrinsic and extrinsic apoptotic pathways," Neuroscience, vol. 135, no. 3, pp. 815-827, 2005.

[16] T. J. Woodward, T. Timic Stamenic, and S. M. Todorovic, "Neonatal general anesthesia causes lasting alterations in excitatory and inhibitory synaptic transmission in the ventrobasal thalamus of adolescent female rats," Neurobiology of Disease, vol. 127, pp. 472-481, 2019.

[17] L. Sun, "Early childhood general anaesthesia exposure and neurocognitive development," British Journal of Anaesthesia, vol. 105, Suppl 1, pp. i61-i68, 2010.

[18] A. Fredriksson, E. Pontén, T. Gordh, and P. Eriksson, "Neonatal exposure to a combination of N-methyl-D-aspartate and gamma-aminobutyric acid type A receptor anesthetic agents potentiates apoptotic neurodegeneration and persistent behavioral deficits," Anesthesiology, vol. 107, no. 3, pp. 427-436, 2007.

[19] J. Hirsch, S. Vacas, N. Terrando et al., "Perioperative cerebrospinal fluid and plasma inflammatory markers after orthopedic surgery," Journal of Neuroinflammation, vol. 13, no. 1, p. 211, 2016

[20] J. Yan, A. Luo, J. Gao et al., "The role of SIRT1 in neuroinflammation and cognitive dysfunction in aged rats after anesthesia and surgery," American Journal of Translational Research, vol. 11, no. 3, pp. 1555-1568, 2019.

[21] Y. Beloosesky, D. Hendel, A. Weiss et al., "Cytokines and Creactive protein production in hip-fracture-operated elderly patients," The Journals of Gerontology. Series A, Biological Sciences and Medical Sciences, vol. 62, no. 4, pp. 420-426, 2007.

[22] A. Buvanendran, J. S. Kroin, R. A. Berger et al., "Upregulation of prostaglandin E2 and interleukins in the central nervous system and peripheral tissue during and after surgery in humans," Anesthesiology, vol. 104, no. 3, pp. 403-410, 2006.

[23] G. Z. Réus, G. R. Fries, L. Stertz et al., "The role of inflammation and microglial activation in the pathophysiology of psychiatric disorders," Neuroscience, vol. 300, pp. 141-154, 2015.

[24] E. Drapeau, W. Mayo, C. Aurousseau, M. Le Moal, P. V. Piazza, and D. N. Abrous, "Spatial memory performances of aged rats in the water maze predict levels of hippocampal neu- rogenesis," Proceedings of the National Academy of Sciences of the United States of America, vol. 100, no. 24, pp. 1438514390, 2003.

[25] R. Sanuki, T. Tanaka, F. Suzuki, K. Ibaraki, and T. Takano, "Normal aging hyperactivates innate immunity and reduces the medical efficacy of minocycline in brain injury," Brain, Behavior, and Immunity, vol. 80, pp. 427-438, 2019.

[26] Y. Zhao, L. Huang, H. Xu et al., "Neuroinflammation induced by surgery does not impair the reference memory of young adult mice," Mediators of Inflammation, vol. 2016, Article ID 3271579, 8 pages, 2016.

[27] J. A. Shin, S. I. Jeong, M. Kim, J. C. Yoon, H. S. Kim, and E. M. Park, "Visceral adipose tissue inflammation is associated with age-related brain changes and ischemic brain damage in aged mice," Brain, Behavior, and Immunity, vol. 50, pp. 221-231, 2015.

[28] A. C. Wendeln, K. Degenhardt, L. Kaurani et al., "Innate immune memory in the brain shapes neurological disease hallmarks," Nature, vol. 556, no. 7701, pp. 332-338, 2018.

[29] X. Liu, D. P. Nemeth, A. J. Tarr et al., "Euflammation attenuates peripheral inflammation-induced neuroinflammation and mitigates immune-to-brain signaling," Brain, Behavior, and Immunity, vol. 54, pp. 140-148, 2016.

[30] Z. M. Qian, X. He, T. Liang et al., "Lipopolysaccharides upregulate hepcidin in neuron via microglia and the IL-6/STAT3 signaling pathway," Molecular Neurobiology, vol. 50, no. 3, pp. 811-820, 2014.

[31] Z. Yu, H. Fukushima, C. Ono et al., "Microglial production of TNF-alpha is a key element of sustained fear memory," Brain, Behavior, and Immunity, vol. 59, pp. 313-321, 2017.

[32] T. H. Ferreira-Vieira, I. M. Guimaraes, F. R. Silva, and F. M. Ribeiro, "Alzheimer's disease: targeting the cholinergic system," Current Neuropharmacology, vol. 14, no. 1, pp. 101115,2016

[33] X. Zhang, X. Jiang, L. Huang et al., "Central cholinergic system mediates working memory deficit induced by anesthesia/surgery in adult mice," Brain and Behavior: A Cognitive Neuroscience Perspective, vol. 8, no. 5, article e00957, 2018. 